\title{
Combating Adversarial Misspellings with Robust Word Recognition
}

\author{
Danish Pruthi Bhuwan Dhingra Zachary C. Lipton \\ Carnegie Mellon University \\ Pittsburgh, USA \\ \{ddanish, bdhingra\}@cs.cmu.edu, zlipton@cmu.edu
}

\begin{abstract}
To combat adversarial spelling mistakes, we propose placing a word recognition model in front of the downstream classifier. Our word recognition models build upon the RNN semicharacter architecture, introducing several new backoff strategies for handling rare and unseen words. Trained to recognize words corrupted by random adds, drops, swaps, and keyboard mistakes, our method achieves $32 \%$ relative (and 3.3\% absolute) error reduction over the vanilla semi-character model. Notably, our pipeline confers robustness on the downstream classifier, outperforming both adversarial training and off-the-shelf spell checkers. Against a BERT model fine-tuned for sentiment analysis, a single adversarially-chosen character attack lowers accuracy from $90.3 \%$ to $45.8 \%$. Our defense restores accuracy to $75 \%{ }^{1}$. Surprisingly, better word recognition does not always entail greater robustness. Our analysis reveals that robustness also depends upon a quantity that we denote the sensitivity.
\end{abstract}

\section{Introduction}

Despite the rapid progress of deep learning techniques on diverse supervised learning tasks, these models remain brittle to subtle shifts in the data distribution. Even when the permissible changes are confined to barely-perceptible perturbations, training robust models remains an open challenge. Following the discovery that imperceptible attacks could cause image recognition models to misclassify examples (Szegedy et al., 2013), a veritable sub-field has emerged in which authors iteratively propose attacks and countermeasures.

For all the interest in adversarial computer vision, these attacks are rarely encountered outside of academic research. However, adversarial

\footnotetext{
${ }^{1}$ All code for our defenses, attacks, and baselines is available at https://github.com/danishpruthi/ Adversarial-Misspellings
}

\begin{tabular}{|c|c|c|}
\hline Alteration & Movie Review & Labe \\
\hline Original & $\begin{array}{l}\text { A triumph, relentless and beautiful } \\
\text { in its downbeat darkness }\end{array}$ & + \\
\hline Swap & $\begin{array}{l}\text { A triumph, relentless and beuatiful } \\
\text { in its downbeat darkness }\end{array}$ & - \\
\hline Drop & $\begin{array}{l}\text { A triumph, relentless and beautiful } \\
\text { in its dwnbeat darkness }\end{array}$ & - \\
\hline+ Defense & $\begin{array}{l}\text { A triumph, relentless and beautiful } \\
\text { in its downbeat darkness }\end{array}$ & + \\
\hline+ Defense & $\begin{array}{l}\text { A triumph, relentless and beautiful } \\
\text { in its downbeat darkness }\end{array}$ & + \\
\hline
\end{tabular}

Table 1: Adversarial spelling mistakes inducing sentiment misclassification and word-recognition defenses.

misspellings constitute a longstanding real-world problem. Spammers continually bombard email servers, subtly misspelling words in efforts to evade spam detection while preserving the emails' intended meaning (Lee and $\mathrm{Ng}, 2005$; Fumera et al., 2006). As another example, programmatic censorship on the Internet has spurred communities to adopt similar methods to communicate surreptitiously (Bitso et al., 2013).

In this paper, we focus on adversarially-chosen spelling mistakes in the context of text classification, addressing the following attack types: dropping, adding, and swapping internal characters within words. These perturbations are inspired by psycholinguistic studies (Rawlinson, 1976; Matt Davis, 2003) which demonstrated that humans can comprehend text altered by jumbling internal characters, provided that the first and last characters of each word remain unperturbed.

First, in experiments addressing both BiLSTM and fine-tuned BERT models, comprising four different input formats: word-only, char-only, word+char, and word-piece (Wu et al., 2016), we demonstrate that an adversary can degrade a classifier's performance to that achieved by random guessing. This requires altering just two charac- 
ters per sentence. Such modifications might flip words either to a different word in the vocabulary or, more often, to the out-of-vocabulary token UNK . Consequently, adversarial edits can degrade a word-level model by transforming the informative words to UNK. Intuitively, one might suspect that word-piece and character-level models would be less susceptible to spelling attacks as they can make use of the residual word context. However, our experiments demonstrate that character and word-piece models are in fact more vulnerable. We show that this is due to the adversary's effective capacity for finer grained manipulations on these models. While against a word-level model, the adversary is mostly limited to UNK -ing words, against a word-piece or character-level model, each character-level add, drop, or swap produces a distinct input, providing the adversary with a greater set of options.

Second, we evaluate first-line techniques including data augmentation and adversarial training, demonstrating that they offer only marginal benefits here, e.g., a BERT model achieving 90.3 accuracy on a sentiment classification task, is degraded to 64.1 by an adversarially-chosen 1character swap in the sentence, which can only be restored to 69.2 by adversarial training.

Third (our primary contribution), we propose a task-agnostic defense, attaching a word recognition model that predicts each word in a sentence given a full sequence of (possibly mispelled) inputs. The word recognition model's outputs comprise the input to a downstream classification model. Our word recognition models build upon the RNN-based semi-character word recognition model due to Sakaguchi et al. (2017). While our word recognizers are trained on domain-specific text from the task at hand, they often predict UNK at test time, owing to the small domain-specific vocabulary. To handle unobserved and rare words, we propose several backoff strategies including falling back on a generic word recognizer trained on a larger corpus. Incorporating our defenses, BERT models subject to 1-character attacks are restored to $88.3,81.1,78.0$ accuracy for swap, drop, add attacks respectively, as compared to $69.2,63.6$, and 50.0 for adversarial training

Fourth, we offer a detailed qualitative analysis, demonstrating that a low word error rate alone is insufficient for a word recognizer to confer robustness on the downstream task. Additionally, we find that it is important that the recognition model supply few degrees of freedom to an attacker. We provide a metric to quantify this notion of sensitivity in word recognition models and study its relation to robustness empirically. Models with low sensitivity and word error rate are most robust.

\section{Related Work}

Several papers address adversarial attacks on NLP systems. Changes to text, whether word- or character-level, are all perceptible, raising some questions about what should rightly be considered an adversarial example (Ebrahimi et al., 2018b; Belinkov and Bisk, 2018). Jia and Liang (2017) address the reading comprehension task, showing that by appending distractor sentences to the end of stories from the SQuAD dataset (Rajpurkar et al., 2016), they could cause models to output incorrect answers. Inspired by this work, Glockner et al. (2018) demonstrate an attack that breaks entailment systems by replacing a single word with either a synonym or its hypernym. Recently, Zhao et al. (2018) investigated the problem of producing natural-seeming adversarial examples, noting that adversarial examples in NLP are often ungrammatical (Li et al., 2016).

In related work on character-level attacks, Ebrahimi et al. (2018b,a) explored gradient-based methods to generate string edits to fool classification and translation systems, respectively. While their focus is on efficient methods for generating adversaries, ours is on improving the worst case adversarial performance. Similarly, Belinkov and Bisk (2018) studied how synthetic and natural noise affects character-level machine translation. They considered structure invariant representations and adversarial training as defenses against such noise. Here, we show that an auxiliary word recognition model, which can be trained on unlabeled data, provides a strong defense.

Spelling correction (Kukich, 1992) is often viewed as a sub-task of grammatical error correction (Ng et al., 2014; Schmaltz et al., 2016). Classic methods rely on a source language model and a noisy channel model to find the most likely correction for a given word (Mays et al., 1991; Brill and Moore, 2000). Recently, neural techniques have been applied to the task (Sakaguchi et al., 2017; Li et al., 2018), which model the context and orthography of the input together. Our work extends the ScRNN model of Sakaguchi et al. (2017). 


\section{Robust Word Recognition}

To tackle character-level adversarial attacks, we introduce a simple two-stage solution, placing a word recognition model $(W)$ before the downstream classifier $(C)$. Under this scheme, all inputs are classified by the composed model $C \circ W$. This modular approach, with $W$ and $C$ trained separately, offers several benefits: (i) we can deploy the same word recognition model for multiple downstream classification tasks/models; and (ii) we can train the word recognition model with larger unlabeled corpora.

Against adversarial mistakes, two important factors govern the robustness of this combined model: $W$ 's accuracy in recognizing misspelled words and $W$ 's sensitivity to adversarial perturbations on the same input. We discuss these aspects in detail below.

\subsection{ScRNN with Backoff}

We now describe semi-character RNNs for word recognition, explain their limitations, and suggest techniques to improve them.

ScRNN Model Inspired by the psycholinguistic studies (Matt Davis, 2003; Rawlinson, 1976), Sakaguchi et al. (2017) proposed a semi-character based RNN ( $\mathrm{ScRNN}$ ) that processes a sentence of words with misspelled characters, predicting the correct words at each step. Let $s=$ $\left\{w_{1}, w_{2}, \ldots, w_{n}\right\}$ denote the input sentence, a sequence of constituent words $w_{i}$. Each input word $\left(w_{i}\right)$ is represented by concatenating (i) a one hot vector of the first character $\left(\mathbf{w}_{\mathbf{i} 1}\right)$; (ii) a one hot representation of the last character $\left(\mathbf{w}_{\mathrm{il}}\right.$, where $l$ is the length of word $w_{i}$ ); and (iii) a bag of characters representation of the internal characters $\left(\sum_{j=2}^{l-1} \mathbf{w}_{\mathbf{i j}}\right)$. ScRNN treats the first and the last characters individually, and is agnostic to the ordering of the internal characters. Each word, represented accordingly, is then fed into a BiLSTM cell. At each sequence step, the training target is the correct corresponding word (output dimension equal to vocabulary size), and the model is optimized with cross-entropy loss.

Backoff Variations While Sakaguchi et al. (2017) demonstrate strong word recognition performance, a drawback of their evaluation setup is that they only attack and evaluate on the subset of words that are a part of their training vocabulary. In such a setting, the word recognition per- formance is unreasonably dependant on the chosen vocabulary size. In principle, one can design models to predict (correctly) only a few chosen words, and ignore the remaining majority and still reach $100 \%$ accuracy. For the adversarial setting, rare and unseen words in the wild are particularly critical, as they provide opportunities for the attackers. A reliable word-recognizer should handle these cases gracefully. Below, we explore different ways to back off when the ScRNN predicts UNK (a frequent outcome for rare and unseen words):

- Pass-through: word-recognizer passes on the (possibly misspelled) word as is.

- Backoff to neutral word: Alternatively, noting that passing UNK-predicted words through unchanged exposes the downstream model to potentially corrupted text, we consider backing off to a neutral word like 'a', which has a similar distribution across classes.

- Backoff to background model: We also consider falling back upon a more generic word recognition model trained upon a larger, less-specialized corpus whenever the foreground word recognition model predicts $\mathrm{UNK}^{2}$. Figure 1 depicts this scenario pictorially.

Empirically, we find that the background model (by itself) is less accurate, because of the large number of words it is trained to predict. Thus, it is best to train a precise foreground model on an in-domain corpus and focus on frequent words, and then to resort to a general-purpose background model for rare and unobserved words. Next, we delineate our second consideration for building robust word-recognizers.

\subsection{Model Sensitivity}

In computer vision, an important factor determining the success of an adversary is the norm constraint on the perturbations allowed to an image $\left(\left\|\mathbf{x}-\mathbf{x}^{\prime}\right\|_{\infty}<\epsilon\right)$. Higher values of $\epsilon$ lead to a higher chance of mis-classification for at least one $\mathrm{x}^{\prime}$. Defense methods such as quantization $(\mathrm{Xu}$ et al., 2017) and thermometer encoding (Buckman et al., 2018) try to reduce the space of perturbations available to the adversary by making the model invariant to small changes in the input.

\footnotetext{
${ }^{2}$ Potentially the background model could be trained with full vocabulary so that it never predicts UNK
} 


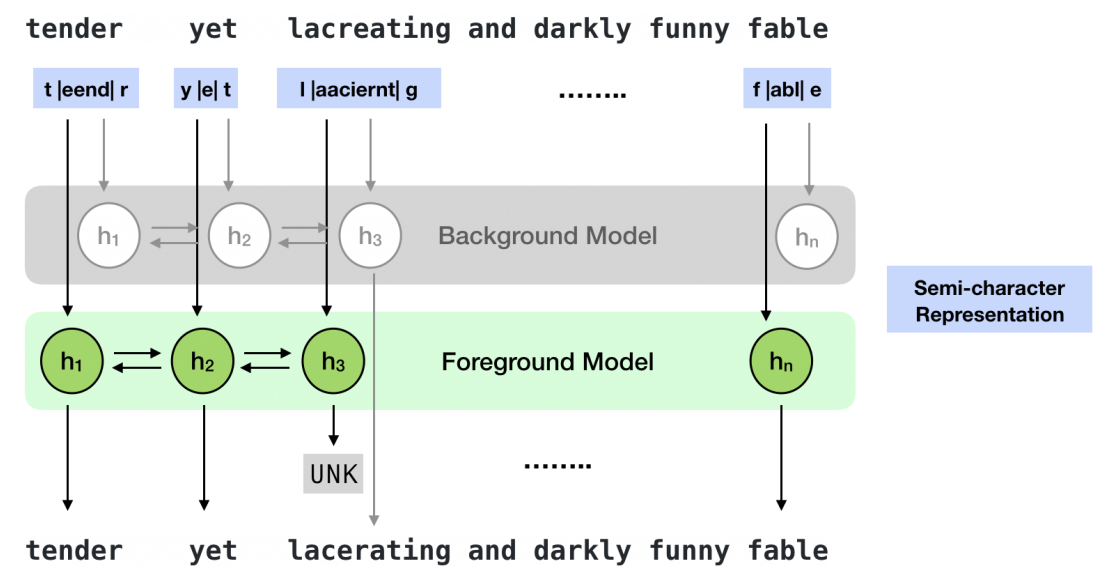

Figure 1: A schematic sketch of our proposed word recognition system, consisting of a foreground and a background model. We train the foreground model on the smaller, domain-specific dataset, and the background model on a larger dataset (e.g., the IMDB movie corpus). We train both models to reconstruct the correct word from the orthography and context of the individual words, using synthetically corrupted inputs during training. Subsequently, we invoke the background model whenever the foreground model predicts UNK .

In NLP, we often get such invariance for free, e.g., for a word-level model, most of the perturbations produced by our character-level adversary lead to an UNK at its input. If the model is robust to the presence of these UNK tokens, there is little room for an adversary to manipulate it. Characterlevel models, on the other hand, despite their superior performance in many tasks, do not enjoy such invariance. This characteristic invariance could be exploited by an attacker. Thus, to limit the number of different inputs to the classifier, we wish to reduce the number of distinct word recognition outputs that an attacker can induce, not just the number of words on which the model is "fooled". We denote this property of a model as its sensitivity.

We can quantify this notion for a word recognition system $W$ as the expected number of unique outputs it assigns to a set of adversarial perturbations. Given a sentence $s$ from the set of sentences $\mathcal{S}$, let $A(s)=s_{1}{ }^{\prime}, s_{2}{ }^{\prime}, \ldots, s_{n}{ }^{\prime}$ denote the set of $n$ perturbations to it under attack type $A$, and let $V$ be the function that maps strings to an input representation for the downstream classifier. For a word level model, $V$ would transform sentences to a sequence of word ids, mapping OOV words to the same UNK ID. Whereas, for a char (or word+char, word-piece) model, $V$ would map inputs to a sequence of character IDs. Formally, sensitivity is defined as

$S_{W, V}^{A}=\mathbb{E}_{s}\left[\frac{\# u\left(V \circ W\left(s_{1}{ }^{\prime}\right), \ldots, V \circ W\left(s_{n}{ }^{\prime}\right)\right)}{n}\right]$, where $V \circ W\left(s_{i}\right)$ returns the input representation (of the downstream classifier) for the output string produced by the word-recognizer $W$ using $s_{i}$ and $\#_{u}(\cdot)$ counts the number of unique arguments.

Intuitively, we expect a high value of $S_{W, V}^{A}$ to lead to a lower robustness of the downstream classifier, since the adversary has more degrees of freedom to attack the classifier. Thus, when using word recognition as a defense, it is prudent to design a low sensitivity system with a low error rate. However, as we will demonstrate, there is often a trade-off between sensitivity and error rate.

\subsection{Synthesizing Adversarial Attacks}

Suppose we are given a classifier $C: \mathcal{S} \rightarrow \mathcal{Y}$ which maps natural language sentences $s \in \mathcal{S}$ to a label from a predefined set $y \in \mathcal{Y}$. An adversary for this classifier is a function $A$ which maps a sentence $s$ to its perturbed versions $\left\{s_{1}^{\prime}, s_{2}^{\prime}, \ldots, s_{n}^{\prime}\right\}$ such that each $s_{i}^{\prime}$ is close to $s$ under some notion of distance between sentences. We define the robustness of classifier $C$ to the adversary $A$ as:

$$
R_{C, A}=\mathbb{E}_{s}\left[\min _{s^{\prime} \in A(s)} \mathbb{1}\left[C\left(s^{\prime}\right)=y\right]\right],
$$

where $y$ represents the ground truth label for $s$. In practice, a real-world adversary may only be able to query the classifier a few times, hence $R_{C, A}$ represents the worst-case adversarial performance of $C$. Methods for generating adversarial examples, such as HotFlip (Ebrahimi et al., 2018b), focus on efficient algorithms for searching the min 
above. Improving $R_{C, A}$ would imply better robustness against all these methods.

Allowed Perturbations $(A(s))$ We explore adversaries which perturb sentences with four types of character-level edits: (1) Swap: swapping two adjacent internal characters of a word. (2) Drop: removing an internal character of a word. (3) Keyboard: substituting an internal character with adjacent characters of QWERTY keyboard (4) Add: inserting a new character internally in a word. In line with the psycholinguistic studies (Matt Davis, 2003; Rawlinson, 1976), to ensure that the perturbations do not affect human ability to comprehend the sentence, we only allow the adversary to edit the internal characters of a word, and not edit stopwords or words shorter than 4 characters.

Attack Strategy For 1-character attacks, we try all possible perturbations listed above until we find an adversary that flips the model prediction. For 2-character attacks, we greedily fix the edit which had the least confidence among 1-character attacks, and then try all the allowed perturbations on the remaining words. Higher order attacks can be performed in a similar manner. The greedy strategy reduces the computation required to obtain higher order attacks ${ }^{3}$, but also means that the robustness score is an upper bound on the true robustness of the classifier.

\section{Experiments and Results}

In this section, we first discuss our experiments on the word recognition systems.

\subsection{Word Error Correction}

Data: We evaluate the spell correctors from $\S 3$ on movie reviews from the Stanford Sentiment Treebank (SST) (Socher et al., 2013). The SST dataset consists of 8544 movie reviews, with a vocabulary of over $16 \mathrm{~K}$ words. As a background corpus, we use the IMDB movie reviews (Maas et al., 2011), which contain $54 \mathrm{~K}$ movie reviews, and a vocabulary of over $78 \mathrm{~K}$ words. The two datasets do not share any reviews in common. The spellcorrection models are evaluated on their ability to correct misspellings. The test setting consists of reviews where each word (with length $\geq 4$, barring stopwords) is attacked by one of the attack types (from swap, add, drop and keyboard at-

\footnotetext{
${ }^{3}$ Its complexity is $O(l)$, instead of $O\left(l^{m}\right)$ where $l$ is the sentence length and $m$ is the order.
}

tacks). In the all attack setting, we mix all attacks by randomly choosing one for each word. This most closely resembles a real world attack setting.

Experimental Setup In addition to our word recognition models, we also compare to After The Deadline (ATD), an open-source spell corrector $^{4}$. We found ATD to be the best freelyavailable corrector ${ }^{5}$. We refer the reader to Sakaguchi et al. (2017) for comparisons of ScRNN to other anonymized commercial spell checkers.

For the ScRNN model, we use a single-layer BiLSTM with a hidden dimension size of 50. The input representation consists of 198 dimensions, which is thrice the number of unique characters (66) in the vocabulary. We cap the vocabulary size to $10 \mathrm{~K}$ words, whereas we use the entire vocabulary of 78470 words when we backoff to the background model. For training these networks, we corrupt the movie reviews according to all attack types, i.e., applying one of the 4 attack types to each word, and trying to reconstruct the original words via cross entropy loss.

Word Recognition

\begin{tabular}{lccccc}
\hline Spell-Corrector & Swap & Drop & Add & Key & All \\
\hline ATD & 7.2 & 12.6 & 13.3 & 6.9 & 11.2 \\
\hline ScRNN $(78 K)$ & 6.3 & 10.2 & 8.7 & 9.8 & 8.7 \\
\hline \multicolumn{5}{c}{ ScRNN $(10 \mathrm{~K})$} & w/ Backoff Variants \\
\hline Pass-Through & 8.5 & 10.5 & 10.7 & 11.2 & 10.2 \\
Neutral & 8.7 & 10.9 & 10.8 & 11.4 & 10.6 \\
Background & $\mathbf{5 . 4}$ & $\mathbf{8 . 1}$ & $\mathbf{6 . 4}$ & $\mathbf{7 . 6}$ & $\mathbf{6 . 9}$ \\
\hline
\end{tabular}

Table 2: Word Error Rates (WER) of ScRNN with each backoff strategy, plus ATD and an ScRNN trained only on the background corpus ( $78 \mathrm{~K}$ vocabulary) The error rates include $5.25 \%$ OOV words.

Results We calculate the word error rates (WER) of each of the models for different attacks and present our findings in Table 2. Note that ATD incorrectly predicts 11.2 words for every 100 words (in the 'all' setting), whereas, all of the backoff variations of the ScRNN reconstruct better. The most accurate variant involves backing off to the background model, resulting in a low error rate of $6.9 \%$, leading to the best performance on word recognition. This is a $32 \%$ relative error

\footnotetext{
${ }^{4}$ https: / / www . afterthedeadline.com/

${ }^{5} \mathrm{We}$ compared ATD with Hunspell (http: //hunspell.github.io/), which is used in Linux applications. ATD was significantly more robust owing to taking context into account while correcting.
} 
reduction compared to the vanilla ScRNN model with a pass-through backoff strategy. We can attribute the improved performance to the fact that there are $5.25 \%$ words in the test corpus that are unseen in the training corpus, and are thus only recoverable by backing off to a larger corpus. Notably, only training on the larger background corpus does worse, at $8.7 \%$, since the distribution of word frequencies is different in the background corpus compared to the foreground corpus.

\subsection{Robustness to adversarial attacks}

We use sentiment analysis and paraphrase detection as downstream tasks, as for these two tasks, 1-2 character edits do not change the output labels.

Experimental Setup For sentiment classification, we systematically study the effect of character-level adversarial attacks on two architectures and four different input formats. The first architecture encodes the input sentence into a sequence of embeddings, which are then sequentially processed by a BiLSTM. The first and last states of the BiLSTM are then used by the softmax layer to predict the sentiment of the input. We consider three input formats for this architecture: (1) Word-only: where the input words are encoded using a lookup table; (2) Char-only: where the input words are encoded using a separate singlelayered BiLSTM over their characters; and (3) Word+Char: where the input words are encoded using a concatenation of (1) and (2) ${ }^{6}$.

The second architecture uses the fine-tuned BERT model (Devlin et al., 2018), with an input format of word-piece tokenization. This model has recently set a new state-of-the-art on several NLP benchmarks, including the sentiment analysis task we consider here. All models are trained and evaluated on the binary version of the sentence-level Stanford Sentiment Treebank (Socher et al., 2013) dataset with only positive and negative reviews.

We also consider the task of paraphrase detection. Here too, we make use of the fine-tuned BERT (Devlin et al., 2018), which is trained and evaluated on the Microsoft Research Paraphrase Corpus (MRPC) (Dolan and Brockett, 2005).

\footnotetext{
${ }^{6}$ Implementation details: The embedding dimension size for the word, char and word+char models are 64, 32 and $64+32$ respectively, with 64,64 and 128 set as the hidden dimension sizes for the three models.
}

Baseline defense strategies Two common methods for dealing with adversarial examples include: (1) data augmentation (DA) (Krizhevsky et al., 2012); and (2) adversarial training (Adv) (Goodfellow et al., 2014). In DA, the trained model is fine-tuned after augmenting the training set with an equal number of examples randomly attacked with a 1-character edit. In Adv, the trained model is fine-tuned with additional adversarial examples (selected at random) that produce incorrect predictions from the current-state classifier. The process is repeated iteratively, generating and adding newer adversarial examples from the updated classifier model, until the adversarial accuracy on dev set stops improving.

Results In Table 3, we examine the robustness of the sentiment models under each attack and defense method. In the absence of any attack or defense, BERT (a word-piece model) performs the best $\left(90.3 \%^{7}\right)$ followed by word+char models $(80.5 \%)$, word-only models $(79.2 \%)$ and then char-only models (70.3\%). However, even singlecharacter attacks (chosen adversarially) can be catastrophic, resulting in a significantly degraded performance of $46 \%, 57 \%, 59 \%$ and $33 \%$, respectively under the 'all' setting.

Intuitively, one might suppose that word-piece and character-level models would be more robust to such attacks given they can make use of the remaining context. However, we find that they are the more susceptible. To see why, note that the word 'beautiful' can only be altered in a few ways for word-only models, either leading to an UNK or an existing vocabulary word, whereas, word-piece and character-only models treat each unique character combination differently. This provides more variations that an attacker can exploit. Following similar reasoning, add and key attacks pose a greater threat than swap and drop attacks. The robustness of different models can be ordered as word-only $>$ word + char $>$ char-only $\sim$ word-piece, and the efficacy of different attacks as add $>$ key $>$ drop $>$ swap.

Next, we scrutinize the effectiveness of defense methods when faced against adversarially chosen attacks. Clearly from table 3, DA and Adv are not

\footnotetext{
${ }^{7}$ The reported accuracy on SST-B by BERT in Glue Benchmarks is slightly higher as it is trained and evaluated on phrase-level sentiment prediction task which has more training examples compared to the sentence-level task we consider. We use the official source code at https: //github.com/google-research/bert
} 
Sentiment Analysis (1-char attack/2-char attack)

\begin{tabular}{|c|c|c|c|c|c|c|}
\hline Model & No attack & Swap & Drop & Add & Key & All \\
\hline \multicolumn{7}{|c|}{ Word-Level Models } \\
\hline BiLSTM & 79.2 & $(64.3 / 53.6)$ & $(63.7 / 52.7)$ & $(60.0 / 43.2)$ & $(60.2 / 42.4)$ & $(58.6 / 40.2)$ \\
\hline BiLSTM + ATD & 79.3 & $(76.2 / 75.3)$ & $(66.5 / 59.9)$ & $(55.6 / 47.5)$ & $(62.6 / 57.6)$ & $(55.8 / 37.0)$ \\
\hline BiLSTM + Pass-through & 79.3 & $(78.6 / 78.5)$ & $(69.1 / 65.3)$ & $(65.0 / 59.2)$ & $(69.6 / 65.6)$ & $(63.2 / 52.4)$ \\
\hline BiLSTM + Background & 78.8 & $(78.9 / 78.4)$ & $(69.6 / 66.8)$ & $(62.6 / 56.4)$ & $(68.2 / 62.2)$ & $(59.6 / 49.0)$ \\
\hline BiLSTM + Neutral & 80.1 & $(80.1 / 79.9)$ & $(72.4 / 70.2)$ & $(67.2 / 61.2)$ & $(69.0 / 64.6)$ & $(63.2 / 54.0)$ \\
\hline \multicolumn{7}{|c|}{ Char-Level Models } \\
\hline BiLSTM & 70.3 & $(53.6 / 42.9)$ & $(48.8 / 37.1)$ & $(33.8 / 14.8)$ & $(40.8 / 22.0)$ & $(32.6 / 14.0)$ \\
\hline BiLSTM + ATD & 71.0 & $(66.6 / 65.2)$ & $(58.0 / 53.0)$ & $(54.6 / 44.4)$ & $(61.6 / 57.5)$ & $(46.5 / 35.4)$ \\
\hline BiLSTM + Pass-through & 70.3 & $(65.8 / 62.9)$ & $(58.3 / 54.2)$ & $(54.0 / 44.2)$ & $(58.8 / 52.4)$ & $(51.6 / 39.8)$ \\
\hline BiLSTM + Background & 70.1 & $(70.3 / 69.8)$ & $(60.4 / 57.7)$ & $(57.4 / 52.6)$ & $(58.8 / 54.2)$ & $(53.6 / 47.2)$ \\
\hline BiLSTM + Neutral & 70.7 & (70.7/70.7) & $(62.1 / 60.5)$ & $(57.8 / 53.6)$ & $(61.4 / 58.0)$ & $(55.2 / 48.4)$ \\
\hline \multicolumn{7}{|c|}{ Word+Char Models } \\
\hline BiLSTM & 80.5 & $(63.9 / 52.3)$ & $(62.8 / 50.8)$ & $(57.8 / 39.8)$ & $(58.4 / 40.8)$ & $(56.6 / 35.6)$ \\
\hline BiLSTM + ATD & 80.8 & $(78.0 / 77.3)$ & $(67.7 / 60.9)$ & $(55.6 / 50.5)$ & $(68.7 / 64.6)$ & $(48.5 / 37.4)$ \\
\hline BiLSTM + Pass-through & 80.1 & (79.0/78.7) & $(69.5 / 65.7)$ & $(64.0 / 59.0)$ & $(66.0 / 62.0)$ & $(61.5 / 56.5)$ \\
\hline BiLSTM + Background & 79.5 & $(79.6 / 79.0)$ & $(69.7 / 66.7)$ & $(62.0 / 57.0)$ & $(65.0 / 56.5)$ & (59.4/49.8) \\
\hline BiLSTM + Neutral & 79.5 & $(79.5 / 79.4)$ & (71.2/68.8) & $(65.0 / 59.0)$ & $(65.5 / 61.5)$ & $(61.5 / 55.5)$ \\
\hline \multicolumn{7}{|c|}{ Word-piece Models } \\
\hline BERT & 90.3 & $(64.1 / 47.4)$ & $(59.2 / 39.9)$ & $(46.2 / 26.4)$ & $(54.3 / 34.9)$ & $(45.8 / 24.6)$ \\
\hline $\mathrm{BERT}+\mathrm{DA}$ & 90.2 & $(68.3 / 50.6)$ & $(62.7 / 39.9)$ & $(43.6 / 17.0)$ & $(57.7 / 32.4)$ & $(41.0 / 15.8)$ \\
\hline $\mathrm{BERT}+\mathrm{Adv}$ & 89.6 & $(69.2 / 52.9)$ & $(63.6 / 40.5)$ & $(50.0 / 22.0)$ & $(60.1 / 36.6)$ & $(47.0 / 20.2)$ \\
\hline BERT + ATD & 89.0 & $(84.5 / 84.5)$ & $(73.0 / 64.0)$ & $(77.0 / 69.5)$ & $(\mathbf{8 0 . 0} / 75.0)$ & $(67.0 / 55.0)$ \\
\hline BERT + Pass-through & 89.8 & $(85.5 / 83.9)$ & $(78.9 / 75.0)$ & $(70.4 / 64.4)$ & $(75.3 / 70.3)$ & $(68.0 / 58.5)$ \\
\hline BERT + Background & 89.3 & (89.1/89.1) & $(79.3 / 76.5)$ & $(76.5 / 71.0)$ & $(77.5 / 74.4)$ & $(73.0 / 67.5)$ \\
\hline BERT + Neutral & 88.3 & $(88.3 / 88.3)$ & $(81.1 / 79.5)$ & (78.0/74.0) & $(78.8 / 76.8)$ & (75.0/68.0) \\
\hline
\end{tabular}

Table 3: Accuracy of various classification models, with and without defenses, under adversarial attacks. Even 1-character attacks significantly degrade classifier performance. Our defenses confer robustness, recovering over $76 \%$ of the original accuracy, under the 'all' setting for all four model classes.

effective in this case. We observed that despite a low training error, these models were not able to generalize to attacks on newer words at test time. ATD spell corrector is the most effective on keyboard attacks, but performs poorly on other attack types, particularly the add attack strategy.

The ScRNN model with pass-through backoff offers better protection, bringing back the adversarial accuracy within $5 \%$ range for the swap attack. It is also effective under other attack classes, and can mitigate the adversarial effect in wordpiece models by $21 \%$, character-only models by $19 \%$, and in word, and word+char models by over $4.5 \%$. This suggests that the direct training signal of word error correction is more effective than the indirect signal of sentiment classification available to DA and Adv for model robustness.

We observe additional gains by using background models as a backoff alternative, because of its lower word error rate (WER), especially, under the swap and drop attacks. However, these gains do not consistently translate in all other settings, as lower WER is necessary but not sufficient. Besides lower error rate, we find that a solid defense should furnish the attacker the fewest options to attack, i.e. it should have a low sensitivity. As we shall see in section $\S 4.3$, the backoff neutral variation has the lowest sensitivity due to mapping UNK predictions to a fixed neutral word. Thus, it results in the highest robustness on most of the attack types for all four model classes.

\begin{tabular}{lccc}
\hline \multirow{2}{*}{ Model } & \multirow{2}{*}{ No Attack } & \multicolumn{2}{c}{ All attacks } \\
\cline { 2 - 4 } & & 1-char & 2-char \\
\hline BERT & 89.0 & 60.0 & 31.0 \\
BERT + ATD & 89.9 & 75.8 & 61.6 \\
BERT + Pass-through & 89.0 & $\mathbf{8 4 . 5}$ & 81.5 \\
BERT + Neutral & 84.0 & 82.5 & $\mathbf{8 2 . 5}$ \\
\hline
\end{tabular}

Table 4: Accuracy of BERT, with and without defenses, on MRPC when attacked under the 'all' attack setting. 
Sensitivity Analysis

\begin{tabular}{lccccc}
\hline Backoff & Swap & Drop & Add & Key & All \\
\hline \multicolumn{5}{c}{ Closed Vocabulary Models (word-only) } \\
\hline Pass-Through & 17.6 & 19.7 & 0.8 & 7.3 & 11.3 \\
Background & 19.5 & 22.3 & 1.1 & 9.5 & 13.1 \\
Neutral & 17.5 & 19.7 & 0.8 & 7.2 & 11.3 \\
\hline \multicolumn{5}{c}{ Open Vocab. Models (char/word+char/word-piece) } \\
\hline Pass-Through & 39.6 & 35.3 & 19.2 & 26.9 & 30.3 \\
Background & 20.7 & 25.1 & 1.3 & 11.6 & 14.7 \\
Neutral & 17.5 & 19.7 & 0.8 & 7.2 & 11.3 \\
\hline
\end{tabular}

Table 5: Sensitivity values for word recognizers. Neutral backoff shows lowest sensitivity.

Table 4 shows the accuracy of BERT on 200 examples from the dev set of the MRPC paraphrase detection task under various attack and defense settings. We re-trained the ScRNN model variants on the MRPC training set for these experiments. Again, we find that simple 1-2 character attacks can bring down the accuracy of BERT significantly ( $89 \%$ to $31 \%$ ). Word recognition models can provide an effective defense, with both our pass-through and neutral variants recovering most of the accuracy. While the neutral backoff model is effective on 2-char attacks, it hurts performance in the no attack setting, since it incorrectly modifies certain correctly spelled entity names. Since the two variants are already effective, we did not train a background model for this task.

\subsection{Understanding Model Sensitivity}

Experimental setup To study model sensitivity, for each sentence, we perturb one randomlychosen word and replace it with all possible perturbations under a given attack type. The resulting set of perturbed sentences is then fed to the word recognizer (whose sensitivity is to be estimated). As described in equation 1, we count the number of unique predictions from the output sentences. Two corrections are considered unique if they are mapped differently by the downstream classifier.

Results The neutral backoff variant has the lowest sensitivity (Table 5). This is expected, as it returns a fixed neutral word whenever the ScRNN predicts an UNK, therefore reducing the number of unique outputs it predicts. Open vocabulary (i.e. char-only, word+char, word-piece) downstream classifiers consider every unique combination of characters differently, whereas wordonly classifiers internally treat all out of vocabulary (OOV) words alike. Hence, for char-only,
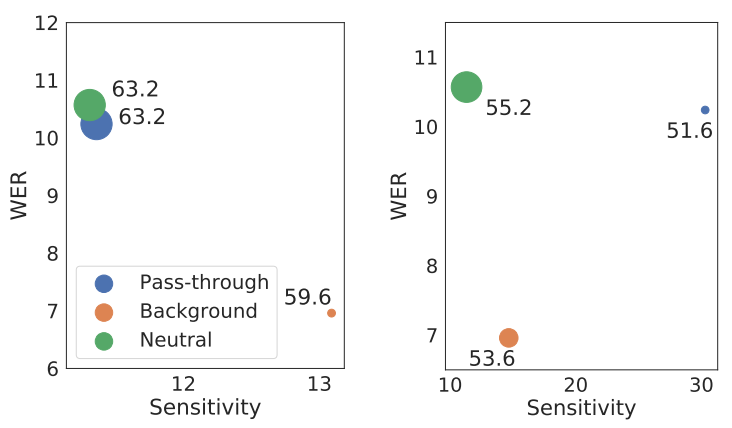

Figure 2: Effect of sensitivity and word error rate on robustness (depicted by the bubble sizes) in word-only models (left) and char-only models (right).

word+char, and word-piece models, the passthrough version is more sensitive than the background variant, as it passes words as is (and each combination is considered uniquely). However, for word-only models, pass-through is less sensitive as all the OOV character combinations are rendered identical.

Ideally, a preferred defense is one with low sensitivity and word error rate. In practice, however, we see that a low error rate often comes at the cost of sensitivity. We visualize this trade-off in Figure 2, where we plot WER and sensitivity on the two axes, and depict the robustness when using different backoff variants. Generally, sensitivity is the more dominant factor out of the two, as the error rates of the considered variants are reasonably low.

Human Intelligibility We verify if the sentiment (of the reviews) is preserved with char-level attacks. In a human study with 50 attacked (and subsequently misclassified), and 50 unchanged reviews, it was noted that 48 and 49 , respectively, preserved the sentiment.

\section{Conclusion}

As character and word-piece inputs become commonplace in modern NLP pipelines, it is worth highlighting the vulnerability they add. We show that minimally-doctored attacks can bring down accuracy of classifiers to random guessing. We recommend word recognition as a safeguard against this and build upon RNN-based semi-character word recognizers. We discover that when used as a defense mechanism, the most accurate word recognition models are not always the most robust against adversarial attacks. Additionally, we highlight the need to control the sensitivity of these models to achieve high robustness. 


\section{Acknowledgements}

The authors are grateful to Graham Neubig, Eduard Hovy, Paul Michel, Mansi Gupta, and Antonios Anastasopoulos for suggestions and feedback.

\section{References}

Yonatan Belinkov and Yonatan Bisk. 2018. Synthetic and natural noise both break neural machine translation. In International Conference on Learning Representations (ICLR).

Constance Bitso, Ina Fourie, and Theo JD Bothma. 2013. Trends in transition from classical censorship to internet censorship: selected country overviews. Innovation: journal of appropriate librarianship and information work in Southern Africa, 2013(46):166-191.

Eric Brill and Robert C. Moore. 2000. An improved error model for noisy channel spelling correction. In Proceedings of the 38th Annual Meeting on Association for Computational Linguistics, ACL '00, pages 286-293, Stroudsburg, PA, USA. Association for Computational Linguistics.

Jacob Buckman, Aurko Roy, Colin Raffel, and Ian Goodfellow. 2018. Thermometer encoding: One hot way to resist adversarial examples. International Conference on Learning Representations (ICLR).

Jacob Devlin, Ming-Wei Chang, Kenton Lee, and Kristina Toutanova. 2018. Bert: Pre-training of deep bidirectional transformers for language understanding. arXiv preprint arXiv:1810.04805.

William B Dolan and Chris Brockett. 2005. Automatically constructing a corpus of sentential paraphrases. In Proceedings of the Third International Workshop on Paraphrasing (IWP2005).

Javid Ebrahimi, Daniel Lowd, and Dejing Dou. 2018a. On adversarial examples for character-level neural machine translation. In International Conference on Computational Linguistics (COLING).

Javid Ebrahimi, Anyi Rao, Daniel Lowd, and Dejing Dou. 2018b. Hotflip: White-box adversarial examples for nlp. In Association for Computational Linguistics (ACL).

Giorgio Fumera, Ignazio Pillai, and Fabio Roli. 2006. Spam filtering based on the analysis of text information embedded into images. Journal of Machine Learning Research (JMLR).

Max Glockner, Vered Shwartz, and Yoav Goldberg. 2018. Breaking nli systems with sentences that require simple lexical inferences. In Association for Computational Linguistics (ACL).
Ian J Goodfellow, Jonathon Shlens, and Christian Szegedy. 2014. Explaining and harnessing adversarial examples. In International Conference on Learning Representations (ICLR).

Robin Jia and Percy Liang. 2017. Adversarial examples for evaluating reading comprehension systems. Empirical Methods in Natural Language Processing (EMNLP).

Alex Krizhevsky, Ilya Sutskever, and Geoffrey E Hinton. 2012. Imagenet classification with deep convolutional neural networks. In Advances in neural information processing systems (NIPS).

Karen Kukich. 1992. Techniques for automatically correcting words in text. Acm Computing Surveys (CSUR), 24(4):377-439.

Honglak Lee and Andrew Y Ng. 2005. Spam deobfuscation using a hidden markov model. In CEAS.

Hao Li, Yang Wang, Xinyu Liu, Zhichao Sheng, and Si Wei. 2018. Spelling error correction using a nested rnn model and pseudo training data. arXiv preprint arXiv:1811.00238.

Jiwei Li, Will Monroe, and Dan Jurafsky. 2016. Understanding neural networks through representation erasure. arXiv preprint arXiv:1612.08220.

Andrew L. Maas, Raymond E. Daly, Peter T. Pham, Dan Huang, Andrew Y. Ng, and Christopher Potts. 2011. Learning word vectors for sentiment analysis. In Association for Computational Linguistics (ACL).

Matt Davis. 2003. Psycholinguistic evidence on scrambled letters in reading. https: / /www.mrc-cbu.cam.ac.uk/ people/matt.davis/cmabridge/.

Eric Mays, Fred J. Damerau, and Robert L. Mercer. 1991. Context based spelling correction. Information Processing \& Management, 27(5):517 - 522.

Hwee Tou Ng, Siew Mei Wu, Ted Briscoe, Christian Hadiwinoto, Raymond Hendy Susanto, and Christopher Bryant. 2014. The conll-2014 shared task on grammatical error correction. In Proceedings of the Eighteenth Conference on Computational Natural Language Learning: Shared Task, pages 1-14.

Pranav Rajpurkar, Jian Zhang, Konstantin Lopyrev, and Percy Liang. 2016. SQuAD: 100,000+ questions for machine comprehension of text. In Empirical Methods in Natural Language Processing (EMNLP).

Graham Ernest Rawlinson. 1976. The significance of letter position in word recognition. Ph.D. thesis, University of Nottingham.

Keisuke Sakaguchi, Kevin Duh, Matt Post, and Benjamin Van Durme. 2017. Robsut wrod reocginiton via semi-character recurrent neural network. In $A s$ sociation for the Advancement of Artificial Intelligence $(A A A I)$. 
Allen Schmaltz, Yoon Kim, Alexander M. Rush, and Stuart Shieber. 2016. Sentence-level grammatical error identification as sequence-to-sequence correction. In Proceedings of the 11th Workshop on Innovative Use of NLP for Building Educational Applications, pages 242-251, San Diego, CA. Association for Computational Linguistics.

Richard Socher, Alex Perelygin, Jean Wu, Jason Chuang, Christopher D Manning, Andrew Ng, and Christopher Potts. 2013. Recursive deep models for semantic compositionality over a sentiment treebank. In Empirical Methods in Natural Language Processing (EMNLP).

Christian Szegedy, Wojciech Zaremba, Ilya Sutskever, Joan Bruna, Dumitru Erhan, Ian Goodfellow, and Rob Fergus. 2013. Intriguing properties of neural networks. arXiv preprint arXiv:1312.6199.

Yonghui Wu, Mike Schuster, Zhifeng Chen, Quoc V Le, Mohammad Norouzi, Wolfgang Macherey, Maxim Krikun, Yuan Cao, Qin Gao, Klaus Macherey, et al. 2016. Google's neural machine translation system: Bridging the gap between human and machine translation. arXiv preprint arXiv:1609.08144.

Weilin Xu, David Evans, and Yanjun Qi. 2017. Feature squeezing: Detecting adversarial examples in deep neural networks. arXiv preprint arXiv:1704.01155.

Zhengli Zhao, Dheeru Dua, and Sameer Singh. 2018. Generating natural adversarial examples. In International Conference on Learning Representations (ICLR). 